\title{
The impact of the Cosmological Constant on the Newtonian Gravity
}

\author{
Engel Roza \\ Stripperwei 1, 5551 ST Valkenswaard, The Netherlands \\ Email: engel.roza@onsbrabantnet.nl
}

\begin{abstract}
Summary
By solving the weak field limit of Einstein's Field Equation including the Cosmological Constant, under the constraint of spherical isotropy, it is shown that, at large cosmological distance, the gravitational force exceeds the one that is predicted by Newton's gravity law, such that it corresponds with Milgrom's MOND hypothesis. However, the resulting prediction that, at extremely large distances, gravity with some spatial periodicity turns on-and-off into antigravity marks a decisive difference.
\end{abstract}

Keywords: Cosmological Constant; MOND; dark matter; antigravity

\section{Introduction}

It is well known that the weak field limit of Einstein's Field Equation corresponds with Newton's gravitation law. As I wish to discuss in this article, this is true as long as Einstein's Cosmological Constant is equal to zero. This implies that a non-zero value of this constant modifies Newton's law. Presently, a non-zero value of this constant is considered being feasible, because it would explain the phenomenon that the Universe is expanding in acceleration rather than with a constant rate such as presumed prior to 1998 [1,2]. It means that the Cosmological Constant embodies the "dark energy", which is seen as the true cause of this phenomenon [3]. If the associated modification of Newton's gravitation law would also be responsible for the excessive orbital velocity of stars at the far end of galaxies, it would be fair to state that the Cosmological Constant would embody "dark matter" as well. This raises the question in how far the modification of Newton's gravitation law due to the Cosmological Constant corresponds with the empirical modification of this law as proposed by Milgrom [4], known as MOND (Modified Newtonian Dynamics), as a substitute for the physical dark matter hypothesis for explaining the flat rotation curves of stars in galaxies. It is my aim to show that the gauge freedom in Einstein's Field Equation allows developing a theoretical basis for heuristic MOND, thereby revealing some unexpected properties and predictions. This requires a particular interpretation of the Cosmological Constant, which will be compared with those described in historical overviews by, for instance, Norton [5] and Harvey and Schucking [6], including (a) Einstein's early erroneous interpretation [5,7], (b) the sourceless fluidal space (vacuum energy) solution $[7,8,9,10]$, and (c) the sourced Schwarzschild-de Sitter metric $[11,12,13,14]$. Moreover, the interpretation will be compared with (d) the one proposed by Meierovich in his non-gauge fourvector solution of Einstein's Equation [15]. The resulting dark matter interpretation will be compared with those of Milgrom [4], Meyerovich [15] and with the one of Verlinde [16,17] in his entropic gravity concept.

To do so, first an outline will be given of the line of thought, the details of which being addressed in an appendix (including a derivation of the constraints for the linearization of Einstein's highly non-linear Field Equation). After that, a comparison will be given between 
the developed theoretical model for modified gravity and the view as usually presented in MOND.

\section{The gravitational wave equation}

Let us start by considering the gravitational wave equation as a consequence of the weak field limit of the Einsteinean Field Equation. The equation reads as,

$G_{\mu v}+\Lambda g_{\mu v}=\frac{8 \pi G}{c^{4}} T_{\mu v} \quad$ with $G_{\mu v}=R_{\mu v}-\frac{1}{2} R g_{\mu v}$

where $T_{\mu \nu}$ is the stress-energy tensor, which describes the energy and the momenta of the source(s) and where $R_{\mu \nu}$ and $R$ are respectively the so-called Ricci tensor and the Ricci scalar. These can be calculated if the metric tensor components $g_{\mu \nu}$ are known $[18,19,20]$. The quantity $\Lambda$ is known as Einstein's Cosmological Constant, $c$ is the light velocity in vacuum and $G$ is the gravitational constant. In the case that a particle under consideration is subject to a central force only, the time-space condition shows a spherical symmetric isotropy. This allows to read the metric elements $g_{i j}$ from a simple line element that can be written as

$\mathrm{d} s^{2}=g_{t t}(r, t) \mathrm{d} q_{0}^{2}+g_{r r}(r, t) \mathrm{d} r^{2}+r^{2} \sin ^{2} \vartheta \mathrm{d} \varphi^{2}+r^{2} \mathrm{~d} \vartheta^{2}$

where $q_{0}=\mathrm{i} c t$ and $\mathrm{i}=\sqrt{-1}$.

It means that the number of metric elements $g_{i j}$ reduce to a few, and that only two of them are time and radial dependent. As is well known, Schwarzschild's solution of Einstein's equation for empty space and $\Lambda=0$, relates the metric components as,

$$
g_{r r} g_{t t}=1
$$

Solving Einstein's equation under adoption of a massive source with pointlike distribution $T_{00}=M c^{2} \delta^{3}(r)$, results in a wave equation with the format (see Appendix A),

$$
-\frac{\partial^{2} g_{t t}}{c^{2} \partial t^{2}}+\frac{1}{r} \frac{\partial^{2}\left(r g_{t t}\right)}{\partial r^{2}}=-\frac{8 \pi G M}{c^{2}} g_{r r} \delta^{3}(r) U(t)
$$

where $U(t)$ is Heaviside's step function.

Its stationary solution [21] under the weak field limit

$$
g_{t t}(r, t)=1+h_{\varphi}(r, t), \text { where }\left|h_{\varphi}(r, t)\right|<<1 \text {, }
$$

is the well-known Newtonian potential, 
$\Phi=-\frac{M G}{r}$, where $h_{\varphi}=\frac{2 \Phi}{c^{2}}$

Note: At first sight it may seem that the Appendix is just common textbook stuff. This is partly true indeed. There are some non-trivial differences, though. A first one is the inclusion of the temporal derivatives of $g_{\mu \mu}$. A second one, more importantly for the remainder of this article, is (later on) the inclusion of the Cosmological Constant into the wave equation, under justification of a spatial range of validity. For these reasons Appendix $A$ is an essential part of this article.

Eq. (4) is the equation of a wave that propagates in the direction of $r$ with a velocity $c$. This equation is identical in format as Maxwell's wave equation for electromagnetism. It proves the causality of gravity.

Let us now memorize that Einstein derived his Field Equation by defining a covariant derivative such that that both the covariant derivatives of the Einstein tensor $G_{\mu \nu}$ and the energy-stress tensor $T_{\mu \nu}$ are zero, i.e.,

$\frac{D}{d q_{\mu}} G_{\mu v}=\frac{D}{d q_{\mu}} T_{\mu v}=0$

Actually, this is a sum of covariant derivatives in Einstein notation, i.e.

$\sum_{\mu=0}^{3} \frac{D}{d q_{\mu}} G_{\mu v}=\sum_{\mu=0}^{3} \frac{D}{d q_{\mu}} T_{\mu v}=0$ for $v=0,1,2,3$

From (8) it is concluded that

$G_{\mu v}+B_{\mu v}=A T_{\mu \nu}$, for $\mu=0,1,2,3$

where $A$ is a scalar constant and where $B_{\mu \nu}$ is a tensor with the particular property that its covariant derivative is zero. Furthermore, because of

$$
\frac{D}{d q_{\mu}} g_{\mu v}=0
$$

i.e., because of the property the covariant derivatives of the metric tensor $g_{\mu \nu}$ are zero, we have,

$B_{\mu v}=\Lambda g_{\mu v}$,

where $\Lambda$ is a scalar constant. With inclusion of this Cosmological Constant $\Lambda$, the wave equation is modified to, 
$-\frac{\partial^{2} g_{t t}}{c^{2} \partial t^{2}}+\frac{1}{r} \frac{\partial^{2}\left(r g_{t t}\right)}{\partial r^{2}}+2 \Lambda=-g_{r r} \frac{8 \pi G T_{t t}}{c^{2}}$

If $T_{t t}$ were a pointlike source $T_{t t}=M c^{2} \delta^{3}(r) U(t)$, where $U(t)$ is Heaviside's step function, the static solution of this equation would be provided by the Schwarzschild-de Sitter metric $[11,12,13,14]$, given by

$g_{t t}(r)=\frac{1}{g_{r r}(r)}=1-\frac{R_{S}}{r}-\frac{\Lambda}{3} r^{2}$, with $\quad R_{S}=\frac{2 M G}{c^{2}}$

Obviously, we meet a problem here, because we cannot separate a weak field $\Phi$ (= gravitational potential) from the metric, because we cannot identify an $r$-domain that justifies the adoption of the constraint (5). However, given the fact that a viable wave function can be obtained for $\Lambda=0$, one might expect that it must be possible to obtain a valid wave equation for a weak field $\Phi$ showing a gradual move from $\Lambda=0$ to $\Lambda \neq 0$. The way out from the problem is the consideration that (12) must be valid both for vacuum with a massive source as well as for vacuum without a source. Hence, where an empty space with $\Lambda=0$ corresponds with virtual sources $T_{\mu \mu}=0$, the vacuum with $\Lambda \neq 0$ is a fluidal space with virtual sources $T_{\mu \mu}=-p \Lambda$, with $g_{\mu \mu}=\left(1,1, r^{2} \sin ^{2} \vartheta, r^{2}\right)$, where $p=c^{4} / 8 \pi G[7,8,9,10]$. This particular stress-energy tensor with equal diagonal elements corresponds with the one for a perfect fluid in thermodynamic equilibrium [21]. Inserting a massive source in this fluid will curve the vacuum to $g_{\mu \mu}=\left(g_{t t}, g_{r r}, r^{2} \sin ^{2} \vartheta, r^{2}\right)$. Therefore, inclusion of the Cosmological Constant $\Lambda$ implies that, under absence of massive sources, Einstein's equation can be satisfied if empty space is given up and is replaced by a space that behaves as a perfect liquid in thermodynamic equilibrium. If a massive pointlike source is inserted into this fluid, deriving a meaningful wave equation is less trivial. As shown in Appendix $A$, this is mainly caused by the loss in symmetry between $g_{t t}$ and $g_{r r}$. Schwarzschild's relationship (3), implies a weak field limit,

$$
h_{t \varphi}=-h_{r \varphi}
$$

while the loss of the relationship implies,

$h_{t \varphi} \neq h_{r \varphi}$.

It also means that it is no longer clear how to relate a Newtonian potential $\Phi$ with the metric components, such as shown by (6). To make things even worse, the consequences in all four metric components $g_{t t}, g_{r r}, g_{\vartheta \vartheta}, g_{\varphi \varphi}$ have to be considered. Nevertheless, it will appear (derived in Appendix A) that the low value of the Cosmological Constant still allows a linearization the equation set within a conditioned spatial range, giving rise to a wave equation with the format,

$-\frac{\partial^{2}}{c^{2} \partial t^{2}}(r \Phi)+\frac{\partial^{2}}{\partial r^{2}}(r \Phi)+\lambda^{2}(r \Phi)=-r \frac{8 \pi G M}{c^{2}} \delta^{3}(r) U(t)$, 
where $\lambda^{2}=2 \Lambda$ and where $\frac{2 \Phi}{c^{2}}=h_{t \varphi}\left(\neq-h_{r \varphi}\right)$.

While this wave equation in the weak field limit is compatible with the behavior of $g_{t t}$, the behavior of $g_{r r}$ is different, but can be readily derived from $g_{t t}$. It appears not fully compatible with the behavior of $g_{\vartheta \vartheta}$ and $g_{\varphi \varphi}$. However, beyond a certain low limit of the spatial variable $r$, it does. A derivation of the spatial range limitations will be shown later in this article.

The main message so far is, that the curving of space-time as a consequence of inserting a massive pointlike source in empty space, like assumed under the Schwarzschild-de Sitter condition, is different from the curving of space-time in the case of inserting a massive source in a fluidal vacuum. Appendix A serves to show the details of the derivation. The static format of the wave equation (15) is a potential field set up by a pointlike source with a format that shows up as a modification of Poisson's equation, such that

$$
\frac{\partial^{2}}{\partial r^{2}}(r \Phi)+\lambda^{2}(r \Phi)=-r \frac{8 \pi G M}{c^{2}} \delta^{3}(r)
$$

This result may seem in conflict with the common perception on the influence of the Cosmological Constant in Newtonian gravity. Like discussed in review articles on its history, such as for instance by Norton [5] and by Harvey and Schucking [6], the resulting Laplace equation should match with (12) such that,

$$
\frac{1}{r} \frac{\partial^{2}}{\partial r^{2}}\left(r \Phi^{\prime}\right)+\lambda^{2}=0
$$

This latter one is compatible with the de Sitter-Schwarzschild metric (13), as can be concluded by identifying $g_{t t}$ as $\Phi^{\prime}$. Equation (16) is different as well from Einstein's early perception in 1917 [7], in which he introduced the Cosmological Constant as

$$
\frac{\partial^{2}}{\partial r^{2}}(r \Phi)-\lambda^{2}(r \Phi)=-r \frac{8 \pi G M}{c^{2}} \delta^{3}(r)
$$

which was shown being erroneous [5,6]. The difference between (16) and the commonly accepted view (17), touches the crux of this article. Where (17) traces back to the solution of Einstein's equation given by de Sitter-Schwarzschild metric, solution (16) traces back to the fluidal space solution. It has to be emphasized here that comparing (16) and (17) is comparing apples with pears, because of the semantic difference between $\Phi^{\prime}$ and $\Phi$. Where $\Phi^{\prime}$ applies to $g_{t t}, \Phi$ applies to $h_{\varphi}$, which is a small deviation on top of $g_{t t}$, as defined by (5). What eq.(16) actually expresses, is a tiny change in the space-time curving on top of the one that is caused by an energetic source. If the source is missing, there would be no curving, neither a change (zero $h_{\varphi}$ ). In that case, (16) simply reduces to the identity $0 \equiv 0$. Where eq. (17) is made up at the cosmological level of the whole universe (modelled as a de 
Sitter space), (16) is made up for a spherical system with a central mass, such as applies to solar systems and galaxies. As we shall demonstrate later in this article, this has an impact on the interpretation of the Cosmological Constant.

Although the fluidal space metric is recognized as a viable solution of Einstein's equation $[6,7,8,9,10]$, it is considered as gravitationally paradoxical [6], because the attracting forces between the fluidal molecules would lead to a collapse of the universe, albeit that many proposals have been put forward for physical mechanisms that would prevent the collapse. Norton [5] refers to scientists as Kelvin (1901), Föpple (1897), Charlier (1908) and Selety (1924). In recent years, though, the fluidal space solution has got a revival in, what is dubbed today, as entropic gravity. This concept is based upon the proposal, put forward in 1999 by Jacobson [22], to reverse the argumentation. Rather than considering the fluidal space as the solution of Einstein's equation, he has proposed to consider Einstein's equation as the result of the fluidal space. By describing the fluidal space as matter in thermodynamic equilibrium, such as embodied in the general gas law, and subsequent evaluating this law under application of the law of the entropic motion of molecules, as independently formulated by Raychauduri [23,24] and Landau [25], and invoking the area law for entropy as formulated by Bekenstein [26] and refined by Hawking [27], Jacobson has been able to derive the full format of Einstein's equation. In a particular interpretation of this approach, published in 2011 and 2016, Verlinde has developed a theory that claims to give an adequate explanation for the origin of dark matter $[16,17]$. This claim has given rise to a lot of interest in the subject of entropic gravity. So, after all, it might well be that the fluidal space concept is less gravitationally paradoxical than originally thought and that it makes sense indeed to accept an elaboration of Einstein's equation based upon the fluidal space concept that results into a modification of Poisson's equation for a spherical system with central mass, like formulated by (16).

As noted before and well known of course, Poisson's equation and its modification, is the static state of a wave equation. From the perspective of classic field theory, a wave equation, can be conceived as the result of an equation of motion derived under application of the action principle from a Lagrangian density L of a scalar field with the generic format

$$
\mathrm{L}=-\frac{1}{2} \partial_{\mu} \Phi \partial^{\mu} \Phi+U(\Phi)+\rho \Phi
$$

where $U(\Phi)$ is the potential energy of the field and where $\rho \Phi$ is the source term. Comparing various fields of energy, we have,

$$
\begin{array}{ll}
U(\Phi)=0 & \text { for electromagnetism. } \\
U(\Phi)=-\lambda^{2} \Phi^{2} / 2 & \text { for this case, } \\
U(\Phi)=\lambda^{2} \Phi^{2} / 2 & \text { for the nuclear forces [28]. }
\end{array}
$$

The non-trivial solutions of (20) in homogeneous format, for the first case and the third case, are respectively, 
$\Phi=\frac{\Phi_{0}}{\lambda r}$ and $\Phi=\Phi_{0} \frac{\exp (-\lambda r)}{\lambda r}$

The first case applies to electromagnetism (for $\Phi_{0}=Q \lambda / 4 \pi \varepsilon_{0}$ ) and Newtonian gravity (for $\left.\Phi_{0}=-M G \lambda\right)$. The third case applies to Proca's generalization of the Maxwellian field [29]. The latter one reduces to the first case if $\lambda \rightarrow 0$, while keeping $\Phi_{0} / \lambda$ constant. Generically, it represents a field with a format that corresponds with the potential as in the case of a shielded electric field (Debije [30]), as well as with Yukawa's proposal [28], to explain the short range of the nuclear force.

A straightforward derivation of a potential function from Einstein's equation has been demonstrated before by Meierovich [15]. His potential function equation [15, eq. 24] is slightly different from the one shown by the homogeneous format of (16). It reads as,

$$
\begin{aligned}
& \frac{\partial}{\partial r}\left\{\frac{\partial \bar{\Phi}}{\partial r}+\frac{2}{r} \bar{\Phi}\right\}+\lambda^{2} \bar{\Phi}=0 \rightarrow \frac{\partial^{2} \bar{\Phi}}{\partial r^{2}}+\frac{2}{r} \frac{\partial \bar{\Phi}}{\partial r}+\left(\lambda^{2}-\frac{2}{r^{2}}\right) \bar{\Phi}=0 \rightarrow \\
& \frac{\partial^{2}}{\partial r^{2}}(r \bar{\Phi})+\left(\lambda^{2}-\frac{2}{r^{2}}\right)(r \bar{\Phi})=0 .
\end{aligned}
$$

Note that the $2 / r^{2}$ term makes the equation different from (16). It has to be emphasized now that the semantics of the function $\bar{\Phi}$ are quite different from those of $\Phi$. The latter $(\Phi)$ applies to the scalar potential set up by a pointlike source particle in a fluid of background energy, where the potential would be zero if there were no source. Where $\Phi$ can be viewed as the scalar component of a fourvector subject to the Lorentz gauge, Meierovich's $\bar{\Phi}$ is defined as a vectorial $\Phi$-analogon of a hypothetical fourvector not subject to the Lorentz gauge. In Meierovich's approach, dark matter acts as a central energetic source that curves space-time, even under absence of any source in the centre, while in our approach dark matter changes the curving of space-time evoked by a central energetic force. In our approach the queer Lagrangean $U(\Phi)$, defined in (20) has been motivated by argumentation, while in Meierovich's approach, the same queer Lagrangean is postulated as an hypothesis [15, eq. (7), under convenient heuristic assumption $a=-1]$.

Let us proceed, after this side-step, on (16). It can be readily be verified that this equation can be satisfied by,

$\Phi=\Phi_{0} \frac{\cos \lambda r+\sin \lambda r}{\lambda r}$

Note that the goniometric shape of this solution is a consequence of the plus sign in front of $\lambda^{2}$. It has to emphasized once more that this expression holds under the classical weak field constraint and the presence of a central source of energy that evokes this field as the tiny variation in the generic spherical metric. Later in this article, the weak field condition and the range of validity will be précised further. The shape (23) will reveal some interesting features. In accordance with the concepts of classical field theory, the field strength can be 
established as the spatial derivative of the potential $\Phi$. Identifying $\Phi_{0} / \lambda$ as $-M G$ and $\lambda$ as a range parameter, we may identify this field strength as a cosmological gravitational acceleration $g$. Let us compare this acceleration with the Newtonian one $g_{N}$.

Hence, from

$$
\begin{aligned}
& \left.g=\frac{d \Phi}{d r}=\frac{\Phi_{0}}{\lambda r^{2}}\{(1-\lambda r) \cos \lambda r+(1+\lambda r) \sin \lambda r)\right\} ; \Phi_{0}=-M G \lambda \rightarrow \\
& \left.g=g_{N}\{(1-\lambda r) \cos \lambda r+(1+\lambda r) \sin \lambda r)\right\} ; g_{N}=\frac{M G}{r^{2}} .
\end{aligned}
$$

Not surprisingly, the gravitational acceleration is affected by the Cosmological Constant $\Lambda=\lambda^{2} / 2$. If $\Lambda=0$, the gravitational acceleration equals the Newtonian one $g_{N}$. Under a positive value of the Cosmological Constant, the gravitational acceleration has a different spatial behavior. This is illustrated in figure 1 , which shows the ratio $g / g_{N}$ as a function of the normalized spatial quantity $\lambda r$. Up to the value $\lambda r=3 \pi / 4, g / g_{N}$ rises monotonously up to the value $g / g_{N}=3.33$. This figure shows that, for relative small values of $r$, the cosmological acceleration behaves similarly as the Newtonian one. Its relative strength over the Newtonian one increases significantly for large values of $r$, although it drops below the Newtonian one at $\lambda r>3.45$. Up to slightly below $\lambda r=3 \pi / 4$, this is, as will be shown, a similar behavior as heuristically implemented in MOND. The effective range is determined by the parameter $\lambda$. It might therefore well be that the cosmological gravity force manifests itself only at cosmological scale. Let us consider its consequence.

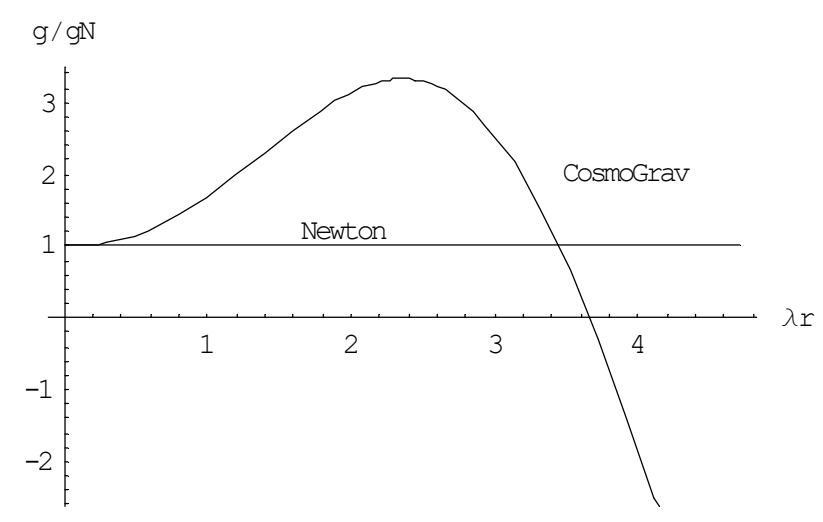

Figure 1: The cosmological gravity force compared with the Newtionian force

Newtonian laws prescribe that the transverse velocity $v_{\varphi}(r)$ of a cosmic object revolving in a circular orbit with radius $r$ in a gravity field is determined by

$$
v_{\varphi}^{2}(r)=\frac{M(r) G}{r} .
$$

where $M(r)$ is the amount of enclosed mass and where $G$ is the gravitational constant. This relationship is often denoted as Kepler's third law. Curiously, like first announced by Vera 
Rubin [31] in 1975, the velocity curve of cosmic objects in a galaxy, such as, for instance, the Milky Way, appears being almost flat. It is tempting to believe that this can be due to a particular spectral distribution of the spectral density to compose $M(r)$. This, however, cannot be true, because $M(r)$ builds up to a constant value of the overall mass. And Kepler's law states in fact that a flat mass curve $M(r)$ is not compatible with a flat velocity curve. Figure 2 illustrates the problem. It is one of the two: either the gravitational acceleration is, at cosmological distances, larger than the Newtonian one, or dark matter, affecting the mass distribution is responsible. Cosmological gravity as expressed by (24) may give the clue. Its effective range is determined by the parameter $\lambda$. It might therefore well be that the cosmological gravity force manifests itself only at cosmological scale. Figure 3 shows that under influence of this force, the rotation curves in the galaxy are subject to a boost.

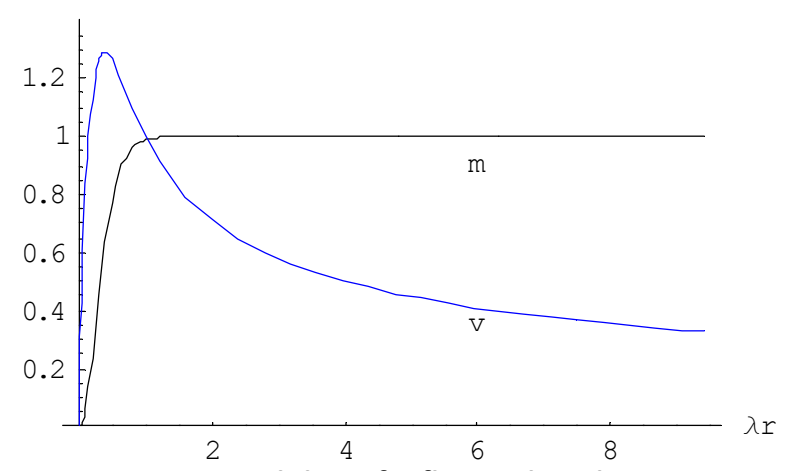

Figure 2. Incompatibility of a flat enclosed mass curve with a flat rotation curve. Note: for illustration purpose a particular distribution is adopted for the enclosed mass. The same distribution is maintained for all subsequent illustrations.

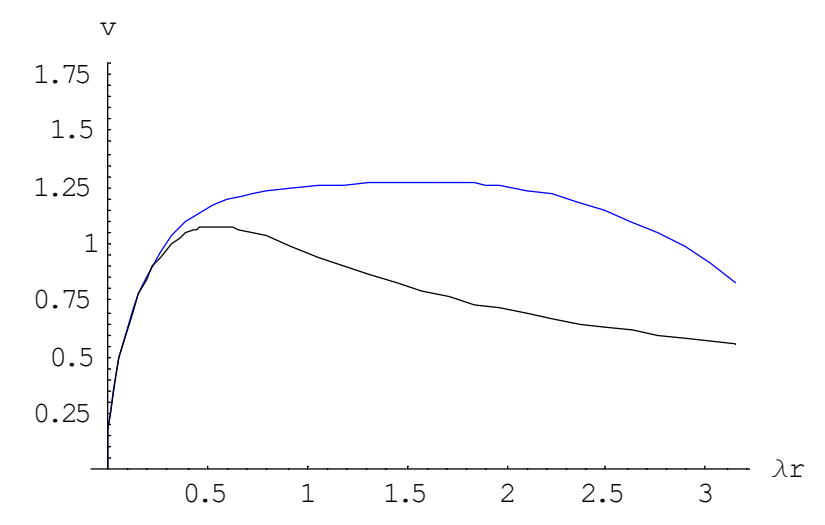

Figure 3: boost of the rotation curve under influence of cosmological gravity.

This cosmological gravity shows another intriguing phenomenon. Like shown in figures 1 and 4 , at the very far cosmological distance, the attraction of gravity is inversed into repulsion. However, rather than the "naked repulsion" (a word used by Harvey and Schucking [6]), like manifest in the de Sitter-Schwarzschild approach, the repulsion shows up at the very far end of the spatial range. Nevertheless, it prevents the clustering of the fluidal space, thereby eliminating the major argument against the fluidal space approach. Further exploration of this phenomenon is a subject outside the scope of this article. It has to be noted that the solution (23) is not unique. There are more solutions possible by modifying the magnitude of $\sin \lambda r$ over $\cos \lambda r$. I have simply chosen here for the symmetrical solution. Cosmological observations would be required to obtain more insight in this. 


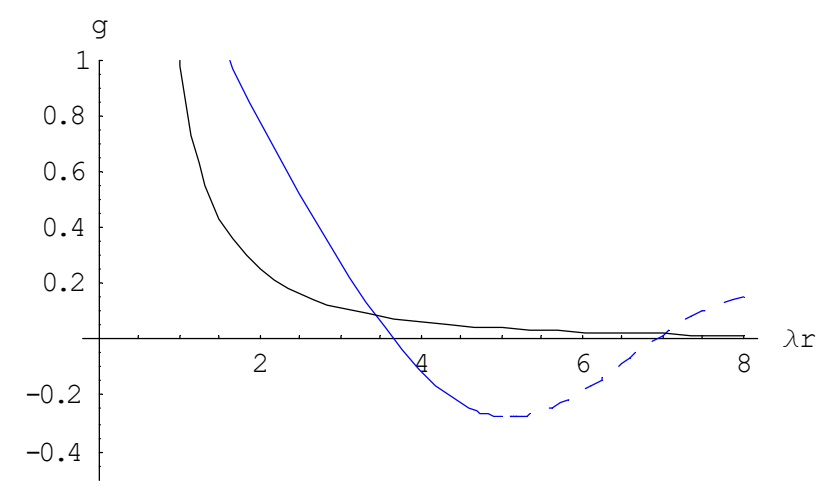

Figure 4: Inversion of the gravity force to antigravity at large cosmological distances. Black: Newtonian. Blue: Cosmological Gravity.

Whether this theoretically derived modification of the Newtonian gravity indeed explains the excessive orbital speeds of stars in a galaxy, such as formulated in Milgrom's empirical law in MOND, is dependent on the numerical value of the Cosmological Constant. The analysis as developed so far in this article gives no clue for calculating this value by theory. However, the theory, as formulated in (23) preserves its value if this quantity, or a related one, can be found from observational evidence, because it might well be that the Cosmological Constant, or a derivate in whatever format, will appear a constant of nature, next to the gravitational constant $G$. The most appropriate way to gain more insight in this, is to compare the modified Newtonian gravity with Milgtom's MOND and to test the theory for galaxies. This will be the subject of the next section.

\section{Comparison with MOND}

MOND is a heuristic approach based on a modification of the gravitational acceleration $g$ such that

$$
g=\frac{g_{N}}{\mu(x)}, \text { with } x=g / a_{0}
$$

where $\mu(x)$ is an interpolation function, $g_{N}\left(=M G / r^{2}\right)$ the Newtonian gravitational acceleration and where $a_{0}$ is an empirical constant acceleration. The format of the interpolation function is not known, but the objectives of MOND are met by a simple function like $[4,33]$

$$
\mu(x)=\frac{x}{\sqrt{1+x^{2}}}
$$

If $g / a_{0}<<1$, such as happens for large $r,(26)$ reduces to

$$
g=\sqrt{a_{0} g_{N}} .
$$

Under this condition, the gravitational acceleration decreases as $r^{-1}$ instead of $r^{-2}$. As a result, the orbital velocity curves as a function of $r$ show up as flat curves.

Algebraic evaluation of (26) and (27) results into, 


$$
\frac{g}{g_{N}}=\sqrt{\frac{1+\sqrt{1+4 k^{2}(\lambda r)^{4}}}{2}} \quad \text { with } k=\frac{a_{0}}{M G \lambda^{2}}
$$

This expression allows a comparison with (24).

As illustrated in figure 5, a pretty good fit is obtained between (24) and (29) in the range up to $\lambda r=3 \pi / 4$ (where the theoretical curve starts decaying), if

$$
k=\frac{a_{0}}{M G \lambda^{2}}=2.5 \rightarrow a_{0}=2.5 M G \lambda^{2}=5 M G \Lambda
$$

Observations on various galaxies have shown that $a_{0}$ can be regarded as a galaxyindependent constant with a value about $a_{0} \approx 1.25 \times 10^{-10} \mathrm{~m} / \mathrm{s}^{2}$.

The implication of (30) is, that $a_{0} \approx 1.25 \times 10^{-10} \mathrm{~m} / \mathrm{s}^{2}$ is a second gravitational constant next to $G$. The two constants determine the range $\lambda$ of the gravitational force in solar systems and galaxy systems as $\lambda^{2} \approx 2 a_{0} / 5 M G$, where $M$ is the enclosed mass in those systems. Where this second gravitational quantity $a_{0}$ is a constant, this is apparently not true for the Einsteinean parameter $\Lambda$.

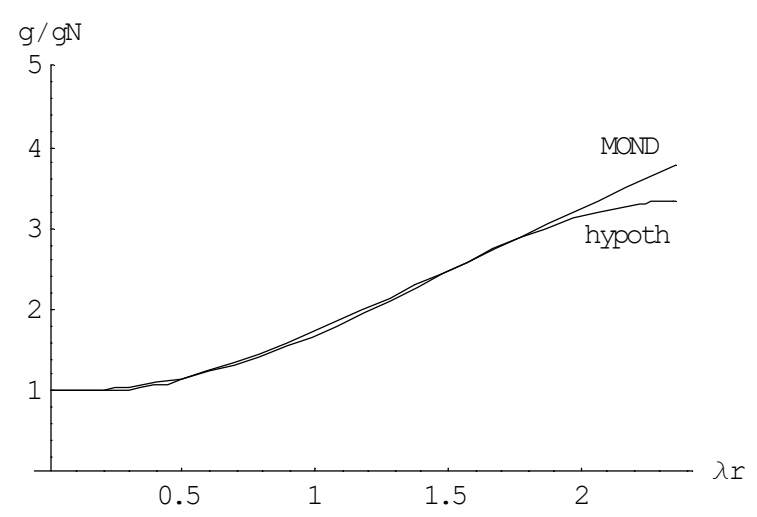

Figure 5: MOND's interpolation function compared with the theory as developed.

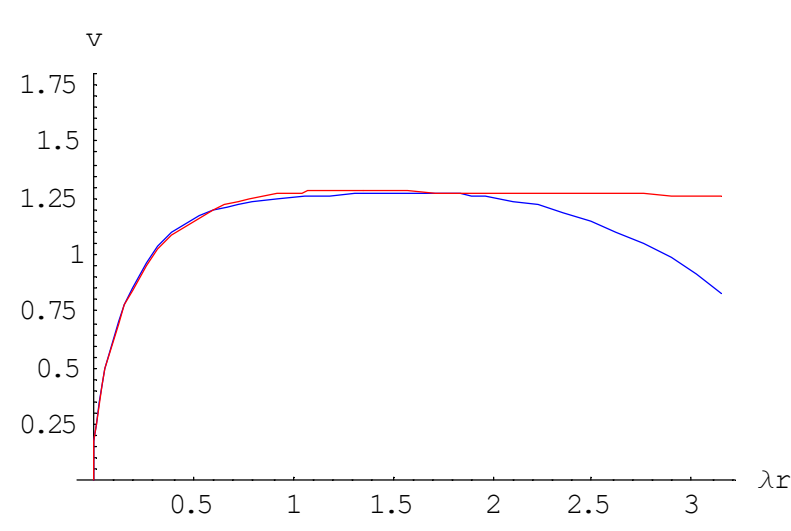

Figure 6: Comparison of orbital velocities for stars in galaxies for MOND (upper curve) and for the theory as developed (lower curve). 
This result shows that Milgom's empirical and the theory as developed in this article are intimately related. Figure 6 shows the difference between curves for the orbital velocity of stars in galaxies according to MOND as compared to those as predicted by the theory as developed in this article. One may ask: "proves MOND the theory or does the theory proves MOND?". Maybe, the better question is: does the well accepted MOND validate the theory? To answer this question, an obvious difference between MOND and the developed theory has to be discussed. From figure 5 it is shown that beyond $r \lambda=3 \pi / 4$ the developed theory deviates from MOND and figure 4 shows that beyond $r \lambda=3.66$ the gravitational attraction changes into a repulsion. From this perspective, the latter phenomenon would even put a natural limit to the size of a galaxy. Let us consider these ranges for the Milky Way. Under the constraint (30), we have

$$
1 / \lambda=\sqrt{5 M G / 2 a_{0}}
$$

The spatial coincidence range between MOND and the theory developed in this article is up to

$$
R_{M}=\frac{3 \pi}{4} \frac{1}{\lambda}=\frac{3 \pi}{4} \sqrt{\frac{2 a_{0}}{5 M G}}=\frac{3 \pi}{4} \sqrt{\frac{4}{5} \frac{a_{0}}{a_{L}} R_{S} L} ; a_{L}=\frac{c^{2}}{L} .
$$

where $R_{S}=2 M G / c^{2}$ is the Schwarzschild radius and $L=c t_{H}$ is the Hubble scale $\left(t_{H}=13.8\right.$ Gyear). Because from calculation $a_{L} \approx 6.9 \times 10^{-10} \mathrm{~m} / \mathrm{s}^{2}$ and because $a_{0} \approx 1.25 \times 10^{-10} \mathrm{~m} / \mathrm{s}^{2}$ from MOND's assessment to most galaxies, if not all, we have from (32) for the Milky Way, where $R_{S} \approx 0.2$ lightyear,

$R_{M} \approx 458000$ lightyear.

This is well beyond the radius of the Milky Way, which amounts to $180.000-200.000$ lightyear. As derived in Appendix A, the coincidence range between MOND and this theory (up to $r \lambda=3 \pi / 4$ ) is well within the spatial validity range $r \lambda<6$ due to the weak field limit constraint and the linearization approximation. Apart from this upper limit for the range of consideration, there is a lower limit as well. This has to do with the weak field limit constraint that we have imposed to derive a single parameter wave equation from Einstein's Field Equation. The value of this lower limit has been derived in Appendix $A$ as

$$
R_{L} \approx\left(2 R_{S} \sqrt{\frac{a_{L}}{a_{0}}} \sqrt{\frac{5 R_{S} L}{2}}\right)^{1 / 2} \rightarrow R_{L} \approx\left(\frac{8}{3 \pi} R_{S} R_{U}\right)^{1 / 2} .
$$

For the Milky Way ( $R_{S} \approx 0.2$ lightyear; $R_{U} \approx 458000$ lightyear) this lower limit amounts to

$R_{L} \approx 279$ lightyear. 
Considering that our solar system is at about 26.000 lightyear from the center, it will be clear that the modified Newtonian gravitation law (24) holds for the major part of the Milky Way. Because many other galaxies are similar to the Milky Way, it is quite probable that this new theory solves the anomaly problem of the stellar rotation problem of most, if not all, galaxies.

\section{Discussion}

In the description given in this article, gravity shows up as the disturbance of the equilibrium state of some fluid in space by a massive source. The fluid executes a negative pressure on the energetic flux from the source. It is the consequence of the adoption of a positive valued cosmological constant in Einstein's Field Equation. How to interpret the physical nature of this fluid is a still unanswered question. Some authors explain the behavior of the fluid as a result of gravitational vacuum polarization [34,35,36,37]. The more beautiful explanation is the reversal of the argumentation, by developing an entropic view, in which Einstein's Field Equation shows up as the result of a thermodynamic modeling of the vacuum, like proposed by Jacobson [18]. In such a view the thermodynamic state of the fluidal gravitational molecules in rest is disturbed by the insertion of an energetic source (baryonic matter), such that energetic level of these molecules is enhanced in a process akin to the Debye process of an electrically charged particle in a plasma. The difference, though, is in the sign of the pressure. In the Debye process, the resulting Coulomb field is suppressed ("screened"), while in the gravitational process the field is enhanced. In such a process the gravitational plasma is polarized under influence of the insertion of a baryonic source, thereby giving rise to the gravitational equivalent of displaced charge. The displaced matter is the true origin of dark matter.

The interpretation of dark matter as displaced matter is somewhat similar, but not identical, to the interpretation proposed by Verlinde in his concept of entropic gravity $[16,17]$. However, where Verlinde considers the dark mass generated by baryonic mass simply as an additive component in Poisson's equation, the Debye view as adopted in this article, prescribes to include in that equation a multiplicative component of dark matter and the gravitational potential, such as become apparent in the static condition of (15). That is the basic reason why the Milgrom effect in Verlinde's result for the gravitational acceleration for small $r$ does not coincide with the Newton acceleration, while it does, and should do, in a true thermodynamic process. To illustrate this further, it is interesting to compare for smal $r$ Milgrom's empirical law with Verlinde's heuristic theory and the result obtained in this article.

Milgrom's result (26) for the gravitational acceleration evolves for $a_{0} / g_{N}<<1$ (i.e., for small $r)$ as

$g=g_{N} \sqrt{\frac{1+\sqrt{1+4\left(\frac{a_{0}}{g_{N}}\right)^{2}}}{2}} \approx g_{N}\left\{1+\frac{1}{2}\left(\frac{a_{o}}{g_{N}}\right)^{2}\right\}$ as long as $\frac{a_{0}}{g_{N}}<<1$,

Verlinde' heuristic theory results into: 
$g=g_{N}\left(1+\sqrt{\frac{a_{0}}{g_{N}}}\right)$.

The theory as developed in this article gives for the gravitational acceleration:

$$
g=\frac{d \Phi}{d r}=\frac{d}{d r}\left\{\Phi_{0}\left(\frac{\cos \lambda r+\sin \lambda r)}{\lambda r}\right\} ; \Phi_{0}=-M G \lambda ; \lambda^{2}=2 \Lambda=\frac{2 a_{0}}{5 M G}\right.
$$

For $a_{0} / g_{N}<<1$, this is approximated as (see Appendix B),

$$
g \approx g_{N}\left(1+\frac{1}{5} \frac{a_{0}}{g_{N}}\right)
$$

To demonstrate the difference between the three expressions, one might consider the gravitational acceleration in Neptune's orbit. In that case $M=1.99 \times 10^{30} \mathrm{~kg}$ (solar mass), $r=4.4910^{12} \mathrm{~m}$ (orbital radius). With $G=6.6710^{-11} \mathrm{~m}^{3} \mathrm{~kg}^{-1} \mathrm{~s}^{-2}$, it makes $g_{N}=0.658 \times 10^{-5}$ $\mathrm{m} / \mathrm{s}^{2}$. This gives for $a_{0}=1.25 \times 10^{-10} \mathrm{~m} / \mathrm{s}^{2}$, respectively,

Milgrom: $g=g_{N}\left(1+1.82 \times 10^{-10}\right) \mathrm{m} / \mathrm{s}^{2}$

Verlinde: $\quad g=g_{N}(1+0.0044) \mathrm{m} / \mathrm{s}^{2}$

this theory: $g=g_{N}\left(1+3.8 \times 10^{-6}\right) \mathrm{m} / \mathrm{s}^{2}$

Unlike the results of Milgrom and those of this theory, the result of Verlinde is considerably beyond the tolerance range prescribed by the gravitational constant $G=6.6710^{-11}(1 \pm 4.7$ $\left.x 10^{-5}\right) \mathrm{m}^{3} \mathrm{~kg}^{-1} \mathrm{~s}^{-2}$. The difference is too large to expect that this large deviation of $G$ in Neptune's orbit would have been unnoticed over time. Milgrom and Sanders have expressed a similar criticism [38]. There are more differences. Unlike Milgrom and Verlinde, this theory predicts that the orbital speed cannot be maintained for extremely large distances from the center of mass. Stated in other words, it means that under some circumstances, dark matter will be missing. Another difference is, that although this explains the excessive orbital speed in galaxies, it is unable to derive by theory a numerical value for Milgrom's acceleration constant. Instead, it proofs that in cosmological systems with a central mass, Milgrom's acceleration is the same if the product of the central mass and the Cosmological Constant is invariant. This means that the Cosmological Constant is not a constant of nature, but a physical quantity proportional with the reciprocal value of the amount of baryonic mass. The true confirmation of Milgrom's numerical value requires an extension of the theory from a system with centric baryonic matter towards the universe with distributed baryonic matter. It can be found in a preprint [39], in which it is shown that this acceleration constant is a true constant of nature, to be assessed by observations. More particularly,

$$
a_{0}=\frac{15}{4} \Omega_{B} a_{L} ; a_{L}=\frac{c^{2}}{\left(c t_{H}\right)}
$$


where $\Omega_{B}$ is the baryonic matter density in the universe, which in the Lamda-CDM model is assessed as $\Omega_{B}=0.0486[40,41]$. With Hubble time scale $t_{H}=13.8 \mathrm{Gyear}$, it gives $a_{0} \approx 1.25$ $\times 10^{-10} \mathrm{~m} / \mathrm{s}^{2}$. Verlinde, though, claims that the $a_{0}=a_{L} / 6$ relationship between Milgrom's acceleration and the Newtonian acceleration at the Hubble scale $\left(a_{0}=a_{L} / 6\right)$ is fundamental (although derived from a heuristic axiomatic principle) and not the consequence of a second gravitational constant next to $G$. All three views (Milgrom, Verlinde, this), however, have in common that dark matter results from a fundamental modification of the Newtonian gravitational law, rather than from an unknown physical substance under preservation of the Newtonian law.

\section{Conclusion}

It has been shown that the weak field limit solution of Einstein's Field Equation with inclusion of the Cosmological Constant $\Lambda$, under the constraint of spherical isotropy, produces a gravitational wave equation with an underlying Lagrangian density in a format that resembles the scalar part of Proca's generalization of the Maxwellian one. Due to the non-linearity of Einstein's equation, the validity range of the equation is constrained between a lower spatial limit and an upper spatial limit. These limits have been quantified and appear being dependent on the product of the Schwarzschild radius of the considered spherical cosmological system and the Cosmological Constant. Because of the match in results, the developed model can be regarded as an underlying theory for the heuristic MOND approach, albeit that the prognosis that, at very large cosmological distances, gravity periodically turns on-and-off into antigravity marks a decisive difference. It is shown in this article that the range determining parameter $\lambda=\sqrt{2 \Lambda}$ is related with a second gravitational constant $a_{0} \approx 1.25 \times 10^{-10} \mathrm{~m} / \mathrm{s}^{2}$ next to $G$. The two constants determine the range $\lambda$ of the gravitational force in solar systems and galaxy systems as $\lambda^{2} \approx 2 a_{0} / 5 M G$, where $M$ is the enclosed mass in those systems. Hence, where this second gravitational quantity $a_{0}$ seems to be a constant of nature, this is not true for the Einsteinean parameter $\Lambda$, which appears being just a scalar constant, i.e., being independent of space-time coordinates. Unlike other theories, where dark matter curves space-time as an independent energetic source next to baryonic sources in empty space, in this theory the dark matter influence on gravity is described as an entropic change, caused by baryonic sources, in the state of a fluidal background energy. The theory as developed in this article gives an adequate explanation for the galaxian phenomenon of flat rotation curves and for the cosmological phenomenon that our universe is expanding in acceleration, such as predicted by Friedmann's law, under influence of a positive value of Einstein's cosmological parameter.

\section{APPENDIX A : THE GRAVITATIONAL WAVE EQUATION}

The objective in this appendix is to derive the weak field limit of the gravitational wave equation with inclusion of the Cosmological Constant. This objective implies that we have to solve Einstein's Field Equation for a spherically symmetric space-time metric that is given by the line element (2), 
$\mathrm{d} s^{2}=g_{t t}(r, t) \mathrm{d} q_{0}^{2}+g_{r r}(r, t) \mathrm{d} r^{2}+r^{2} \sin ^{2} \vartheta \mathrm{d} \varphi^{2}+r^{2} \mathrm{~d} \vartheta^{2}$

where $q_{0}=\mathrm{i} c t$.

Note: The space-time (ict, $r, \vartheta, \varphi$ ) is described on the basis of the "Hawking" metric $(+,+,+,+)$. The components $g_{\mu \mu}$ compose the metric tensor $g_{\mu \nu}$, which determine the Ricci tensor $R_{\mu \nu}$ and the Ricci scalar $R$. These quantities play a decisive role in Einstein's Field Equation, which reads as

$G_{\mu \nu}+\Lambda g_{\mu \nu}=\frac{8 \pi G}{c^{4}} T_{\mu \nu} \quad$ with $G_{\mu \nu}=R_{\mu \nu}-\frac{1}{2} R g_{\mu \nu}$

In a space without massive sources, the Einstein Field Equation under this symmetric spherical isotropy, reduces to a simple set of equations for the elements $R_{\mu \mu}$ of the Ricci tensor,

$$
\begin{array}{ll}
R_{t t}-\frac{1}{2} R g_{t t}+\Lambda g_{t t}=0 ; & R_{r r}-\frac{1}{2} R g_{r r}+\Lambda g_{r r}=0 ; \\
R_{\vartheta \vartheta}-\frac{1}{2} R g_{\vartheta \vartheta}+\Lambda g_{\vartheta \vartheta}=0 ; & R_{\varphi \varphi}-\frac{1}{2} R g_{\varphi \varphi}+\Lambda g_{\varphi \varphi}=0 .
\end{array}
$$

Let us proceed by considering the Ricci scalar. It is defined generically as

$R=\sum_{\mu=0}^{3} \sum_{v=0}^{3} g^{\mu v} R_{\mu v}$.

In spherical symmetry the matrices contain diagonal elements only, so that $(A-4)$ reduces to

$$
R=\sum_{\mu=0}^{3} g^{\mu \mu} R_{\mu \mu} .
$$

This result can be applied to (A-3). Multiplying the first one with $g^{00}\left(=g^{t t}\right)$, the second one with $g^{11}$, etc., and subsequent addition results of the terms $\mu=1,2,3$ gives,

$$
\begin{aligned}
& \sum_{\mu=1}^{3} g^{\mu \mu} R_{\mu \mu}-\frac{3}{2} R+3 \Lambda=-g^{t t} R_{t t}+\sum_{\mu=0}^{3} g^{\mu \mu} R_{\mu \mu}-\frac{3}{2} R+3 \Lambda=-g^{t t} R_{t t}-\frac{1}{2} R+3 \Lambda=0 \\
& \text { hence } g^{t t} R_{t t}=-\frac{1}{2} R+3 \Lambda
\end{aligned}
$$

Repeating this recipe for $g_{\mu \mu}\left(=1 / g^{\mu \mu}\right)$, we have for reasons of symmetry

$$
g^{\mu \mu} R_{\mu \mu}=-\frac{1}{2} R+3 \Lambda .
$$


Note that the subscripts and superscripts $00,11,22$, and 33 are, respectively, identical to $t t, r r, \vartheta \vartheta$ and $\varphi \varphi$. Applying this result to Einstein's equation set gives,

$2 g^{\mu \mu} R_{\mu \mu}-2 \Lambda=\frac{8 \pi G T_{\mu \mu} g^{\mu \mu}}{c^{4}}$,

such that after multiplication by $g_{\mu \mu}$, we have

$2 R_{\mu \mu}-2 g_{\mu \mu} \Lambda=\frac{8 \pi G T_{\mu \mu}}{c^{4}}$,

Let us first proceed under the conditions of the absence of massive sources $\left(T_{\mu \mu}=0\right)$ and let us consider the Ricci tensor components $R_{t t}$ and $R_{r r}$ under use of the results shown in Table A-1, obtained by a calculation shown later in this Appendix. Note: $g^{\prime}$ and $g^{\prime \prime}$ means differentiation, respectively double differentiation of $g$ into $r ; \dot{g}$ and $\ddot{g}$ means differentiation, respectively double differentiation of $g$ into $t$. Multiplying (A-3a) by $1 / g_{t t}$ and $(\mathrm{A}-3 \mathrm{~b})$ by $1 / g_{r r}$ gives,

$\frac{R_{t t}}{g_{t t}}-\Lambda=0$ and $\frac{R_{r r}}{g_{r r}}-\Lambda=0$

which, under the assumption of a zero Cosmological Constant $(\Lambda=0)$, after subtraction and under use of the expressions in Table A-1 results into.

$-\frac{1}{r} \frac{1}{g_{r r}}\left(\frac{g_{r r}^{\prime}}{g_{r r}}+\frac{g_{t t}^{\prime}}{g_{t t}}\right)=0$

Hence

$\frac{g_{r r}^{\prime}}{g_{r r}}+\frac{g_{t t}^{\prime}}{g_{t t}}=0$

which can be integrated to (the Schwarzschild condition),

$g_{r r} g_{t t}=1$

This, in turn, gives

$\frac{\dot{g}_{r r}}{g_{r r}}+\frac{\dot{g}_{t t}}{g_{t t}}=0$

Using (A-13), (A-15) and the Table A-1 values on $R_{t t}$ gives 


$$
R_{t t}=\frac{1}{g_{r r}}\left(-\frac{1}{2} g_{t t}^{\prime \prime}-\frac{1}{r} g_{t t}^{\prime}-\frac{\ddot{g}_{r r}}{2 c^{2}}\right)=\frac{1}{g_{r r}}\left(-\frac{1}{2 r} \frac{\partial^{2}\left(r g_{t t}\right)}{\partial r^{2}}+\frac{1}{2 c^{2}} \frac{\partial^{2} g_{t t}}{\partial t^{2}}\right) .
$$

Hence, from (A-10) and (A-16),

$$
\frac{2}{g_{r r}}\left(-\frac{1}{2 r} \frac{\partial^{2}\left(r g_{t t}\right)}{\partial r^{2}}+\frac{1}{2 c^{2}} \frac{\partial^{2} g_{t t}}{\partial t^{2}}\right)=\frac{8 \pi G T_{t t}}{c^{4}}
$$

or, equivalently,

$$
-\frac{1}{r} \frac{\partial^{2}\left(r g_{t t}\right)}{\partial r^{2}}+\frac{1}{c^{2}} \frac{\partial^{2} g_{t t}}{\partial t^{2}}=\frac{8 \pi G T_{t t}}{c^{4}} g_{r r}
$$

Applying well-known conditions,

$$
\begin{array}{ll}
\Lambda=0 \quad \text { (already assumed) } & \text { (no cosmological constant), } \\
g_{t t}(r, t)=1+h_{\varphi}(r, t), \text { where }\left|h_{\varphi}(r, t)\right|<<1 & \text { (the weak field limit) } \\
T_{t t}=M c^{2} \delta^{3}(r), & \text { (pointlike massive source) }
\end{array}
$$

yields the proper wave equation

$\frac{\partial^{2}\left(r h_{\varphi}\right)}{\partial r^{2}}-\frac{1}{c^{2}} \frac{\partial^{2} r h_{\varphi}}{\partial t^{2}}=-\frac{8 \pi G M}{c^{2}} r \delta^{3}(r) U(t)$,

where $U(t)$ is Heaviside's step function. In the static regime, the equation results into

$$
\frac{1}{r} \frac{\partial^{2}\left(r h_{\varphi}\right)}{\partial r^{2}}=-\frac{8 \pi G M}{c^{2}} \delta^{3}(r)
$$

This is similar to Poisson's equation,

$$
\nabla^{2} \Phi=\frac{1}{r} \frac{\partial^{2}(r \Phi)}{\partial r^{2}}=-4 \pi G \rho=-4 \pi G M \delta^{3}(r)
$$

the solution of which is the Newtonian potential,

$$
\Phi=-\frac{G M}{r}\left[\mathrm{~m}^{2} \mathrm{~s}^{-2}\right]
$$

Comparing (A-20) with (A-22) gives the equivalence 
$h_{\varphi}=\frac{2 \Phi}{c^{2}}$

Let us now consider the case $\Lambda \neq 0$ under absence of a massive source. Obviously, $(A-10)$ is only satisfied if the influence of the cosmological constant is counter balanced by the hypothetical source

$T_{t t}=-p \Lambda$, where $p=\frac{c^{4}}{8 \pi G}$

Because all four members of the Einstein set (A-10) have to be satisfied, we have, under consideration of $(A-10)$ and Table $A 1$,

$T_{\mu \mu}=-p g_{\mu \mu} \Lambda$ and $g_{\mu \mu}=\left(1,1, r^{2} \sin ^{2} \vartheta, r^{2}\right)$

This particular stress-energy tensor with equal diagonal elements corresponds with the one for a perfect fluid in thermodynamic equilibrium [8]. So, where empty space corresponds with virtual sources $T_{\mu \mu}=0$, the fluidal space corresponds with virtual sources $T_{\mu \mu}=-p \Lambda$, with $g_{\mu \mu}=\left(1,1, r^{2} \sin ^{2} \vartheta, r^{2}\right)$. Insertion of a massive pointlike source in this fluid and modifying (A-17) by adding the virtual sources, after redefining the weak limit condition as,

$g_{t t}(r, t)=1+h_{t \varphi}(r, t) ; g_{t r}(r, t)=1+h_{r \varphi}(r, t) ;\left|h_{t \varphi}\right|,\left|h_{r \varphi}\right|<<1$,

gives, for the static parts,

$$
\begin{aligned}
& \left.\frac{2}{g_{r r}}\left\{-\frac{1}{2} h_{t \varphi}^{\prime \prime}+\frac{h_{t \varphi}^{\prime}}{4}\left(h_{r \varphi}^{\prime}+h_{t \varphi}^{\prime}\right)-\frac{1}{r} h_{t \varphi}^{\prime}\right)\right\}-2 \Lambda g_{t t}=\frac{8 \pi G T_{t t}}{c^{4}}-2 \Lambda \rightarrow \\
& -h_{t \varphi}^{\prime \prime}-2 \Lambda h_{t \varphi}+\frac{h_{t \varphi}^{\prime}}{2}\left(h_{r \varphi}^{\prime}+h_{t \varphi}^{\prime}\right)-\frac{2}{r} h_{t \varphi}^{\prime}=\frac{8 \pi G T_{t t}}{c^{4}} g_{r r}
\end{aligned}
$$

and, secondly,

$$
-h_{t \varphi}^{\prime \prime}-2 \Lambda h_{r \varphi}+\frac{h_{t \varphi}^{\prime}}{2}\left(h_{r \varphi}^{\prime}+h_{t \varphi}^{\prime}\right)+\frac{2}{r} h_{r \varphi}^{\prime}=0
$$

As long as $\Lambda=0$, and assuming a pointlike source embodied in $T_{t t}$, the Schwarzschild condition shows up. This is obvious by subtracting the latter equation from the former one, thereby allowing exclusion of a singularity at $r=0$. It reveals that under this condition the homogeneous formats of the two equations are identical. However, because this is no longer true for $\Lambda \neq 0$, we have to cope with two equations. These two equations are non-linear. However, because $h_{t \varphi}$ and $h_{r \varphi}$ are small in the weak field limit, the two equations can be linearized under the condition that the last term in the left-hand part of these equations is dominant over their preceding terms. This assumption, to be checked later, allows to rewite (A-26) for $r \neq 0$, as 


$$
\left(r h_{t \varphi}\right)^{\prime \prime}+2 \Lambda\left(r h_{t \varphi}\right)=0
$$

A simple format for the second equation is obtained after subtraction (A-27) from (A-26), resulting into,

$-2 h_{r \varphi}^{\prime}+2 \Lambda r h_{r \varphi}=2 h_{t \varphi}^{\prime}+2 \Lambda r h_{t \varphi}$.

Obviously, $h_{r \varphi}$ can be calculated as soon as $h_{t \varphi}$ is found as a solution of (A-28). Re-inserting the pointlike source, similarly as in the case $\Lambda=0$ and including the time derivatives, yield a wave equation as a generalization of (A-28). After rewriting,

$$
h_{t \varphi}=\frac{2 \Phi}{c^{2}} \text { and } \Lambda=\frac{\lambda^{2}}{2},
$$

we have from (A-28) the inhomogeneous generalization,

$$
-\frac{\partial^{2}}{c^{2} \partial t^{2}}(r \Phi)+\frac{\partial^{2}}{\partial r^{2}}(r \Phi)+\lambda^{2}(r \Phi)=-r \frac{8 \pi G M}{c^{2}} \delta^{3}(r) U(t)
$$

If $\Lambda<0$, we have under static conditions, a similarity with Helmholtz' equation [42] with the screened Poisson's equation, the solution of which is Yukawa's potential,

$$
\Phi=\frac{G M}{r} \exp (-\lambda r)
$$

which reduces to Poisson's one for $\lambda \rightarrow 0$.

If $\Lambda>0$, we have under static conditions, a similarity with Helmholtz' equation with a characteristic solution,

$\Phi=\frac{G M}{r}\{\cos \lambda r+\sin \lambda r\}$

This solution reduces to Poisson's one for $\lambda \rightarrow 0$ as well.

This is the weak field limit solution of Einstein's Equation if one does not take the validity of Poisson's equation of gravity for granted, but adopts Helmholtz equation instead under an appropriate choice of the Cosmological Constant.

We are not done yet. There are two remaining issues. The first one is the justification of the linearization approximation by moving from (A-26) to (A-28). Moreover, we have to take into consideration that, although the derived gravitational potential field satisfies (A-3a) and (A$3 b)$, we are not sure that it satisfies $(A-3 c)$ and $(A-3 d)$ as well. It should do, to prevent violation of the metric $(A-1)$. Assessment of it is the second thing to be done. 
The remaining issues: (a) the linearization approximation

The linearity approximation $(A-26) \rightarrow(A-28)$ is justified as long as

$\left|\frac{2}{r} h_{t \varphi}^{\prime}\right| \gg>\left|\frac{h_{t \varphi}^{\prime}}{2}\left(h_{r \varphi}^{\prime}+h_{t \varphi}^{\prime}\right)\right| \rightarrow \frac{1}{r}>>\frac{1}{4}\left|h_{r \varphi}^{\prime}+h_{t \varphi}^{\prime}\right|$.

Under consideration of (A-29), it can be written as,

$\frac{1}{\Lambda r^{2}}>>\frac{1}{4}\left|h_{r \varphi}-h_{t \varphi}\right|$

This condition enforces calculation of $h_{r \varphi}$ from $h_{t \varphi}$. From (A-29),

$h_{r \varphi}^{\prime}-\frac{\lambda^{2} r}{2} h_{r \varphi}=-\left(h_{t \varphi}^{\prime}+\frac{\lambda^{2} r}{2} h_{t \varphi}\right)$

This first order differential equation for $h_{r \varphi}$ can be readily solved, albeit that the resulting analytical expression from the generic solution

$h_{r \varphi}(\lambda r)=\frac{1}{p(\lambda r)} \int p(\lambda r) f(\lambda r) d(\lambda r)$, where

$f(\lambda r)=\frac{\partial}{\partial(\lambda r)} h_{t \varphi}(\lambda r)+\frac{\lambda r}{2} h_{t \varphi}(\lambda r) ; p(\lambda r)=\exp \left\{\int-\frac{\lambda r}{2} d(\lambda r)\right\}=\exp \left(-\frac{\lambda^{2} r^{2}}{4}\right)$,

is a rather complicated one.

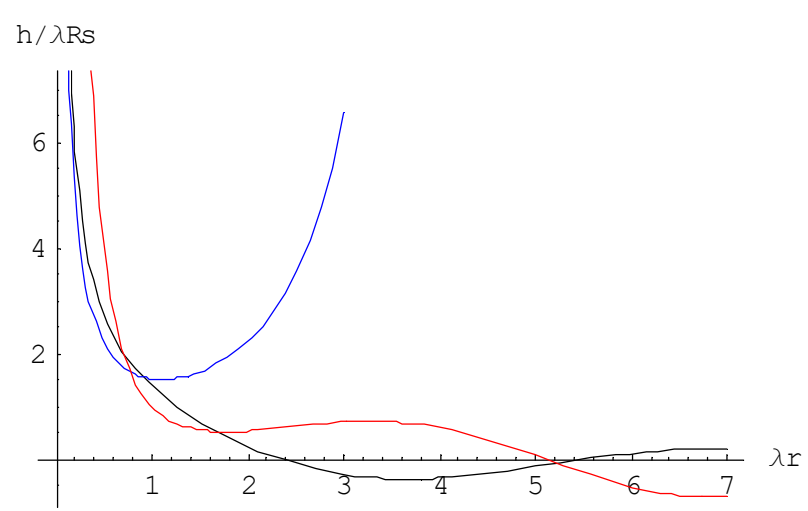

Fig. A-1: Relative values of the metric quantities $h_{t \varphi}$ (black) and $-h_{r \varphi}$ (blue) as a function of $\lambda r$. The red curve represents the function in the right-hand part of (A-36).

Figure A-1 illustrates the behavior of the calculated $-h_{r \varphi}$ as a function of $\lambda r$ compared with $h_{t \varphi}$. From (A-36), it is obvious that if $\lambda r \rightarrow 0, h_{r \varphi} \approx-h_{t \varphi}$. The vertical axis is normalized to a dimensionless quantity, by writing, under consideration of (A-30) and (A-33), 
$h_{t \varphi}=\frac{2}{c^{2}} \frac{G M}{r}\{\cos \lambda r+\sin \lambda r\}=\frac{\lambda R_{S}}{\lambda r} \cos \lambda r+\sin \lambda r ; R_{S}=\frac{2 M G}{c^{2}}$.

Note that $R_{S}$ is the Schwarzschild radius of the cosmological system (with central force) under consideration. The black curve shows the normalized value of $h_{t \varphi}$. It is gradually decreasing by $r^{-1}$. The blue curve shows the normalized value of $h_{r \varphi}$ as calculated from the differential equation (A-36). This quantity tend to explode with increasing $\lambda r$. Nevertheless the functions on both sides of (A-36) remain the same and show the gradual finite behavior, shown by the red curve. The reason is due to sign differences between the left-hand part and the right-hand part of (A-35). Subtraction of two large quantities makes the result still small enough. Nevertheless, the exponential increase of $h_{r \varphi}$ may violate the linearization approximation. This requires proper investigation. Because eventually (for relatively large $\lambda r) h_{r \varphi} \gg h_{t \varphi}$, and considering that $2 \Lambda=\lambda^{2}$, we may reformulate (A-35) as,

$\frac{1}{\lambda r}>>\frac{\lambda}{8} R_{S}\left(\frac{h_{r \varphi}}{\lambda R_{S}}\right)$

Hence, the cross over value $\lambda r_{0}$ is determined as,

$\lambda r_{0}=\frac{8}{h_{r \varphi}\left(r_{0}\right)}=\frac{8}{\lambda R_{S} h_{r \varphi}\left(\lambda r_{0}\right)}$

where $h_{r \varphi}(\lambda r)$ is given by (A-37). From (A-39) it is obvious that as long as $h_{r \varphi}<<1$, the upper limit is far beyond $\lambda r=8$. However, because of the exponential growth of $h_{r \varphi} / \lambda R_{S}$ with $\lambda r$, the limit can be shifted near to this limit or even shifted below. This may spoil the weak field limit assumption. Hence, the actual validity range of the linearization heavily depends on the value of the product $\lambda R_{S}$. Once this product is known, the cross-over value of $\lambda r_{0}$ and the associated value of the metric component $h_{r \varphi}$ can be calculated from the known curve $h_{r \varphi} / \lambda R_{S}$ shown in fig. A-1. Because of its exponential growth, the upper limit for $\lambda r$ that justifies the linearization approximation, is below, but probably near, to the cross-over value.

The assessment of a meaningful quantitative value to the product $\lambda R_{S}$ is possible by invoking the value of the Cosmological Constant for cosmological systems with a central mass. As shown in the main text, this is obtained by the application of the theory to Milgrom's MOND, expressed by eq. (26). This expression relates Einstein's Cosmological Constant $\Lambda$ with Milgrom's acceleration constant $a_{0}$ as,

$2 \Lambda=\lambda^{2}=\frac{2 a_{0}}{5 M G}$.

Hence, 


$$
\lambda R_{S}=2 \sqrt{\frac{a_{0}}{a_{L}} \frac{R_{S}}{L}} ; a_{L}=\frac{c^{2}}{L},
$$

where $a_{L}$ is the gravitational acceleration constant at distance $L$ from the centre of the cosmological system under consideration. Choosing $L$ as the Hubble range $L=c t_{H}$ and defining $c^{2} / L=a_{L}$ as the acceleration at the verge of the Hubble range, and considering that Milgrom's acceleration constant amounts to $a_{0} \approx 1.25 \times 10^{-10} \mathrm{~m} / \mathrm{s}^{2}$, the ratio $a_{L} / a_{0}$ amounts to, $a_{L} / a_{0} \approx 6.9 / 1.25=5.52$. The Schwarzschild radius $R_{S}$ of a typical galaxy, like the Milky Way, is about 0.2 lightyear, while the Hubble time amounts to $t_{H} \approx 13.5$ Gyear. Hence, typically

$$
\lambda R_{S} \approx 3.3 \times 10^{-6}
$$

From (A-42), (A-40) and (A-37), it is found that the cross-over $\lambda r_{0}$ amounts to

$$
\lambda r_{0} \approx 7.25 \text {. }
$$

The associated value of the metric component amounts to $h_{r \varphi}=1.01$. That violates the weak field approximation. However, at $\lambda r=6$, the metric component is drops to $h_{r \varphi}=0.017$. Hence, It is fair to say that, up to a normalized spatial distance near to $\lambda r_{0} \approx 6$, the derived gravitational wave equation (A-31) for galaxies akin to the Milky Way maintains its validity.

The remaining issues: (b) the other two equations

Before appearance of the massive source, we have for (A-3c),

$\left\{1+\frac{r}{2 g_{r r}}\left(\frac{g_{r r}^{\prime}}{g_{r r}}-\frac{g_{t t}^{\prime}}{g_{t t}}\right)-\frac{1}{g_{r r}}\right\}-g_{\vartheta \vartheta} \Lambda=$ background matter,

where $g_{\mu \mu}=\left(1,1, r^{2} \sin ^{2} \vartheta, r^{2}\right)$

After appearance of the massive source, we have

$1+\frac{r}{2 g_{r r}}\left(\frac{g_{r r}^{\prime}}{g_{r r}}-\frac{g_{t t}^{\prime}}{g_{t t}}\right)-\frac{1}{g_{r r}}-g_{\vartheta \vartheta} \Lambda=$ background matter plus source,

where $g_{\mu \mu} \neq\left(1,1, r^{2} \sin ^{2} \vartheta, r^{2}\right)$.

Due to the change of curving by the source, we have,

$$
1+\frac{r}{2 g_{r r}}\left(\frac{g_{r r}^{\prime}}{g_{r r}}-\frac{g_{t t}^{\prime}}{g_{t t}}\right)-\frac{1}{g_{r r}}-\left(g_{\vartheta \vartheta}-r^{2}\right) \Lambda=0 .
$$


Under the constraint of the weak field limit, this equation can be rewritten as,

$$
\Lambda g_{\vartheta \vartheta}=\Lambda r^{2}-r h_{t \varphi}^{\prime}-h_{t \varphi}+\frac{r}{2}\left(h_{r \varphi}^{\prime}+h_{t \varphi}^{\prime}\right)+\left(h_{r \varphi}+h_{t \varphi}\right)=0 .
$$

As long as $\Lambda g_{\vartheta \vartheta}$ is close to $\Lambda r^{2}$, the metric (A-1) maintains it validity. This is true as long as

$$
\begin{aligned}
& r^{2}>>\frac{1}{\Lambda}\left(r h_{t \varphi}^{\prime}+h_{t \varphi}\right), \text { and } \\
& r^{2} \gg>\frac{1}{\Lambda}\left\{\frac{r}{2}\left(h_{r \varphi}^{\prime}+h_{t \varphi}^{\prime}\right)+\left(h_{r \varphi}+h_{t \varphi}\right)\right\} .
\end{aligned}
$$

The split into two conditions is made for ease of analysis. Under consideration of (A-29), (A48b) can be rewritten as,

$$
r^{2}>>\frac{1}{\Lambda}\left\{\left(1+\frac{\lambda^{2} r^{2}}{2}\right) h_{r \varphi}+\left(1-\frac{\lambda^{2} r^{2}}{2}\right) h_{t \varphi}\right\}
$$

Because with increasing $\lambda r$ the quantity $h_{r \varphi}$ is dominating over $h_{t \varphi},(\mathrm{A}-50)$ can be replaced by,

$$
r^{2}>>\frac{1}{\Lambda}\left(\frac{\lambda^{2} r^{2}}{2}\right) h_{r \varphi} \rightarrow r^{2}>>r^{2} h_{r \varphi} \rightarrow h_{r \varphi}<<1
$$

thereby concluding that the condition $(A-49 b)$ is covered by the weak field constraint.

Now we have established the upper spatial limit justified the linearization condition and concluded that condition ( $A-48 \mathrm{~b})$ is covered by the weak field constraint, we are left with a single issue. That is condition (A-48a). Considering that,

$$
\begin{aligned}
& h_{t \varphi}=\frac{2}{c^{2}} \frac{G M}{r}\{\cos \lambda r+\sin \lambda r\} ; h_{t \varphi}^{\prime}=-\frac{R_{S}}{r^{2}}\{\cos \lambda r+\sin \lambda r\}+\frac{R_{S}}{r}(-\lambda \sin \lambda r+\lambda \cos \lambda r) \\
& h_{t \varphi}^{\prime}=-\frac{R_{S}}{r^{2}}\{(\lambda r-1) \cos \lambda r-(1+\lambda r) \sin \lambda r\},
\end{aligned}
$$

we have for (A-49a),

$$
\begin{aligned}
& r^{2}>>\frac{R_{S}}{\Lambda}(-\lambda \sin \lambda r+\lambda \cos \lambda r) \rightarrow \\
& r^{2}>>\frac{2 R_{S}}{\lambda}(-\sin \lambda r+\cos \lambda r) \approx r^{2}+\frac{2\left|R_{S}\right|}{\lambda}=r^{2}+\frac{2\left|R_{S}\right|}{\sqrt{2 a_{0}}} c \sqrt{5 M G / c^{2}}= \\
& r^{2}>>\frac{2\left|R_{S}\right|}{\sqrt{2 a_{0}}} c \sqrt{\frac{5 R_{S}}{2}}=\frac{2\left|R_{S}\right|}{\sqrt{2 a_{0}}} \sqrt{\frac{c^{2}}{L}} \sqrt{\frac{5 R_{S}}{2}} \sqrt{L}=2\left|R_{S}\right| \sqrt{\frac{a_{L}}{a_{0}}} \sqrt{\frac{5 R_{S} L}{2}} .
\end{aligned}
$$


Hence,

$r>>R_{G}$, where $R_{G}=\left(2\left|R_{S}\right| \sqrt{\frac{a_{L}}{a_{0}}} \sqrt{\frac{5 R_{S} L}{2}}\right)^{1 / 2}$.

As already noted, the Schwarzschild radius $R_{S}$ of a typical galaxy, like the Milky Way, is about 0.2 lightyear. For such a galaxy, the range $R_{G}$ calculated from (A-54) appears being $R_{G} \approx 278$ lightyear. Considering that the radius of the Milky Way is estimated as $100.000-180.000$ lightyear and that our solar system is at about 26.000 lightyear from the center, it will be clear that the wave equation (A-31) holds for the major part of the galaxy, thereby solving the anomaly problem of the stellar rotation problem.

Table A1: metric tensor and Ricci tensor

\begin{tabular}{|l|l|}
\hline metric tensor & Ricci tensor \\
\hline$g_{t t} \equiv g_{00}$ & $R_{t t}=-\frac{1}{2} \frac{g_{t t}^{\prime \prime}}{g_{r r}}-\frac{\ddot{g}_{r r}}{2 c^{2} g_{r r}}+\frac{g_{t t}^{\prime}}{4 g_{r r}}\left(\frac{g_{r r}^{\prime}}{g_{r r}}+\frac{g_{t t}^{\prime}}{g_{t t}}\right)-\frac{\dot{g}_{r r}}{4 c^{2} g_{r r}}\left(\frac{\dot{g}_{r r}}{g_{r r}}+\frac{\dot{g}_{t t}}{g_{t t}}\right)-\frac{1}{r} \frac{g_{t t}^{\prime}}{g_{r r}}$ \\
\hline$g_{r r} \equiv g_{11}$ & $R_{r r}=-\frac{1}{2} \frac{g_{t t}^{\prime \prime}}{g_{t t}}-\frac{\ddot{g}_{r r}}{2 c^{2} g_{t t}}+\frac{g_{t t}^{\prime}}{4 g_{t t}}\left(\frac{g_{r r}^{\prime}}{g_{r r}}+\frac{g_{t t}^{\prime}}{g_{t t}}\right)-\frac{\dot{g}_{r r}}{4 c^{2} g_{t t}}\left(\frac{\dot{g}_{r r}}{g_{r r}}+\frac{\dot{g}_{t t}}{g_{t t}}\right)+\frac{1}{r} \frac{g_{r r}^{\prime}}{g_{r r}}$ \\
\hline$g_{\vartheta \vartheta} \equiv g_{22}=r^{2}$ & $R_{\vartheta \vartheta}=1+\frac{r}{2 g_{r r}}\left(\frac{g_{r r}^{\prime}}{g_{r r}}-\frac{g_{t t}^{\prime}}{g_{t t}}\right)-\frac{1}{g_{r r}}$ \\
\hline$g_{\varphi \varphi} \equiv g_{33}=r^{2} \sin ^{2}(\vartheta)$ & $R_{\kappa \varphi}=\sin ^{2}(\vartheta) R_{\vartheta \vartheta}$ \\
\hline
\end{tabular}

\section{APPENDIX B}

$$
\begin{aligned}
& g=\frac{d \Phi}{d r}=\frac{d}{d r}\left\{-\Phi_{0}\left(\frac{\cos \lambda r+\sin \lambda r)}{\lambda r}\right\}=\frac{\Phi_{0}}{\lambda r^{2}}(\cos \lambda r+\sin \lambda r)-\frac{\Phi_{0}}{\lambda r}(-\lambda \sin \lambda r+\lambda \cos \lambda r) \approx\right. \\
& \approx \frac{\Phi_{0}}{\lambda r^{2}}\left(1-\frac{\lambda^{2} r^{2}}{2}+\lambda r\right)+\frac{\Phi_{0}}{\lambda r}\left(\lambda^{2} r-\lambda+\frac{\lambda^{2} r^{2}}{2}\right) \approx \frac{\Phi_{0}}{\lambda r^{2}}\left(1+\frac{1}{2} \lambda^{2} r^{2}\right) .
\end{aligned}
$$

Inserting $\Phi_{0}=M G \lambda, \lambda^{2}=2 a_{0} / 5 M G, g_{N}=M G / r^{2}$, gives $g \approx g_{N}\left(1+\frac{1}{5} \frac{a_{0}}{g_{N}}\right)$. q.e.d.

\section{References}

[1] J.A. Frieman, M.S. Turner, D. Huterer, Dragan, Ann. Rev. Astronomy and Astrophys. 46, 385 (2008)

[2] G. Goldhaber, AIP Conference Proceedings 1166, 53, arXiv:0907.3526 (2009) $^{2}$ 
[3]P.J.E. Peebles, B. Ratra, Bharat (2003). Reviews of Modern Physics. 75 (2): 559, (2003)

[4] M. Milgrom, The Astrophysical Journal, 270, 365 (1983)

[5] J.D. Norton, The Cosmological Woes of Newtonian Gravitation Theory, Einstein studies, 7 . Birkauser, Boston, 217, ISBN 0817640606 (1999)

[6] A. Harvey, E.. Schucking, Am. Journ. of Physics, Vol. 68, Issue 8, 723 (2000).

[7] A. Einstein, Preuss. Akad. Wiss, Berlin (Math. Phys.), 142 (1917)

[8] www.scholarpedia.org/article/Cosmological constant

[9] S. Carroll, W. Press and E. Turner, Ann. Rev. Astronomy and Astrophys, 30, 499 (1992)

[10] J. Sola, Journal of Physics, Conf. Series, 453, 012015 (2013)

[11] F. Kottler, Ann. Physik 56, 361, 401 (1918)

[12] H. Weyl, Phys. Z. 20, 31 (1919)

[13] E. Treffitz, Mathem. Ann. 86, 317

[14] Li-Feng Sun et al., Modern Phys. Lett. A 28, 1350114 (2013)

[15] B. E. Meierovich, Phys. Rev. D, 87, 103510; arXiv:1303.7062v1 [gr-qc], (2013)

[16] E. Verlinde, JHEP 1104, 029 ; arXiv: 1001.0785v1 [hep-th] (2011)

[17] E. Verlinde, SciPost Phys. 2, no. 3, 016 (2017); arXiv:1611.02269v2 [hep-th] (2016)

[18] A. Einstein, Relativity: The Special and General Theory, H. Holt and Company, New York (1916, translation 1920)

[19] S. Weinberg, Gravitation and Cosmology, John Wiley \& Sons, Inc., New York (1972)

[20] T.A. Moore, A General Relativity Workbook, University Science Books, (2013)

[21] B. Schutz, A First Course in General Relativity, $2^{\text {nd }}$ ed, Cambridge Univ. Press, New York (2009)

[22] T. Jacobson, Phys.Rev. Lett., 116, no. 20, 201101 (2016)

[23] A. Raychauduri, Phys.Rev. 106, 172 (1957)

[24] S. Kar, Pramana, Vol. 69, Issue 1, 49 (2008)

[25] M.O. Tahim, R.R. Landim, C.A.S. Almeida, Modern Phys. Letters A, 24, No.15, 1209 (2009);

arXiv: 0705.4120v1 (2007)

[26] J.D. Bekenstein, Phys. Rev. D7, 2333 (1973)

[27] S.W. Hawking, Comm. Math. Phys. 43, 199 (1975)

[28]H. Yukawa, Proc. Phys. Math. Soc. Jpn 17, 48 (1935); H. Yukawa and S. Sakata, Proc. Phys. Math. Soc. Jpn., 19, 1084 (1937)

[29] A. Proca, J. Phys. Radium, 7, 346 (1936); Acad. Sci. Paris, C.R. 202, 1366 (1936)

[30]P. Debye and E. Huckel, Physik. Zetschrift, vol. 24, 9, 185 (1923)

[31] V. Rubin, N. Thonnard, W.K. Ford Jr., Astrophysical Journal, 238, 471 (1980)

[32] A.V. Minkevich, Acta Physica Polonica Series B, 38, 1 (2006)

[33] G. Gentile, B. Famae and W.G.J . de Blok, J. Astronomy and Astrophysics, 527, 76 (2011)

[34] L. Blanchet, Classical and Quantum Gravity, Vol.24, No.14, 3529 (2007)

[35] E.S. Corchero, arXiv:0802.0392 [gr-qc] (2008)

[36] D. Hajdukovic, Astrophysics and Space Science, 334, vol.2, 215 (2011)

[37] S. Rahmati, https:www.uleth.ca/dspace/handle/10133/3302, (2012)

[38] M. Milgrom and R.H. Sanders, arXiv:1612.09582 (2017)

[39] E. Roza, doi:10.20944/preprints201712.0077.v2 (2017)

[40] Planck Collaboration, Astronomy and Astrophys., 594: A13 (2015)

[41] https://en.wikipedia.org/wiki/Lamda-CDM model (april 2018)

[42] M. Abramowitz and I. Stegun eds., Handbook of Mathematical functions with Formulas, Graphs and Mathematical Tables, National Bureau of Standards. Washington, D. C., 1964. 